\title{
3-dimensional electrode patterning within a microfluidic channel using metal ion implantation
}

\author{
Jae-Woo Choi, ${ }^{* a b}$ Samuel Rosset, ${ }^{a}$ Muhamed Niklaus, ${ }^{a}$ James R. Adleman, ${ }^{b}$ Herbert Shea ${ }^{a}$ \\ and Demetri Psaltis ${ }^{a}$
}

\author{
Received 28th August 2009, Accepted 3rd December 2009 \\ First published as an Advance Article on the web 7th January 2010 \\ DOI: $10.1039 / b 917719 a$
}

\begin{abstract}
The application of electrical fields within a microfluidic channel enables many forms of manipulation necessary for lab-on-a-chip devices. Patterning electrodes inside the microfluidic channel generally requires multi-step optical lithography. Here, we utilize an ion-implantation process to pattern 3D electrodes within a fluidic channel made of polydimethylsiloxane (PDMS). Electrode structuring within the channel is achieved by ion implantation at a $40^{\circ}$ angle with a metal shadow mask. The advantages of three-dimensional structuring of electrodes within a fluidic channel over traditional planar electrode designs are discussed. Two possible applications are presented: asymmetric particles can be aligned in any of the three axial dimensions with electro-orientation; colloidal focusing and concentration within a fluidic channel can be achieved through dielectrophoresis. Demonstrations are shown with E. coli, a rod shaped bacteria, and indicate the potential that ion-implanted microfluidic channels have for manipulations in the context of lab-on-a-chip devices.
\end{abstract}

\section{Introduction}

Controlling particles within liquids is critical in many lab-ona-chip applications. ${ }^{\mathbf{1} 2}$ For most applications, an external force is applied to accurately and reliably control a biological cell through the microfluidic channel. Such control has been demonstrated utilizing mechanical, electrical, thermal and optical force methods. ${ }^{3-10}$ Electrical methods require the integration of electrodes to the microfluidic chip. If an electric field is applied between two electrodes on the same plane, a nonuniform electric field distribution is formed within the channel and the directionality and adaptability of the field is limited. Electrodes on all walls of microfluidic channels would be very beneficial to generate an electric field of any alignment for dielectrophoretic and electro-orientation purposes. ${ }^{11,12}$

Dielectrophoresis and electro-orientation allows for the manipulation and differentiation of different samples of interest through size, dielectric properties and size asymmetry. ${ }^{13}$ Recent studies of dielectrophoresis show its capability to separate different beads based on size and determine the dielectric properties of biological samples such as yeast, blood cells and bacteria. ${ }^{11-16}$ Recent studies of electro-orientation have revealed its ability to orient bacteria and nanowires. ${ }^{17-19}$ Dielectrophoresis provides for concentration and sorting capabilities ${ }^{11,12,16}$ and electro-orientation allows for the alignment of asymmetric particles on a macro scale. ${ }^{20}$

Several methods for integration of electrodes with microfluidic channels have been developed. Electrodes forming the channel were demonstrated using highly doped silicon. ${ }^{21}$ Electrodes on the walls of an elliptical microfluidic channel were demonstrated

${ }^{a}$ School of Engineering, Ecole Polytechnique Fédérale de Lausanne (EPFL), Lausanne, 1015, Switzerland.E-mail: jae-woo.choi@epfl.ch

${ }^{b}$ Department of Electrical Engineering, California Institute of Technology, Pasadena, CA, 91125, USA using multi-step optical lithography involving glass wet etching and metal deposition. ${ }^{22}$ Vertical electrodes in the sidewall of a rectangular micro- or nano-fluidic channel were demonstrated using multi-step optical lithography involving SU-8 with metal deposition and electroplating. ${ }^{12,23}$ Single-step optical lithographic fabrication of microfluidic channels has been demonstrated $^{24}$ and simplicity in fabrication of electrodes has been demonstrated as well. ${ }^{25}$ In this paper, we demonstrate a novel technique for the fabrication of electrodes in all four walls of the channel without increasing the complexity of its fabrication.

Electrodes are implanted onto the sidewall and topwall of the PDMS microfluidic channel through an ion-implanting process. ${ }^{26,27}$ The electrodes are connected across the borders by implanting into the PDMS at an angle and exhibit optical transparency over $35 \% .{ }^{27}$ Potential applications utilizing these electrodes within microfluidic channels are demonstrated by aligning and concentrating $E$. coli using electro-orientation and dielectrophoresis.

\section{Design}

A rectangular microfluidic channel $100 \mu \mathrm{m}$ in width, $70 \mu \mathrm{m}$ in height, and approximately $2 \mathrm{~cm}$ in length is fabricated using the replica molding technique. A master mold is produced utilizing UV lithography with SU-8 (GM-1070, Gerstelec) on a silicon wafer. After trimethylchlorosilane (Sigma Aldrich) treatment of the master mold for $5 \mathrm{~min}$, the PDMS (Sylgard 184, Dow Corning) is poured onto the mold with a $10: 1$ base-to-curing agent ratio. After curing in an oven at $80^{\circ} \mathrm{C}$ for $1 \mathrm{~h}$, the silicone is released from the mold. Next, we use low energy metal ion implantation from a Filtered Cathode Voltage Arc (FCVA) source to coat the inside of the microfluidic channel with gold ions through a steel shadow mask. The steel shadow mask consists of two openings of $8 \mathrm{~mm}$ by $15 \mathrm{~mm}$ separated by $100 \mu \mathrm{m}$. 
The ions form a layer of gold nanoparticles in the top $50 \mathrm{~nm}$ of the PDMS. The main advantage of the implantation process is the excellent adhesion of the gold electrodes to the PDMS, since they are implanted a few nanometers below the surface. This adhesion can be verified by using scotch tape on the surface of a PDMS surface with both sputtered and implanted gold. The scotch tape will readily remove the sputtered gold from the PDMS surface while no noticeable change will occur to the implanted gold. The FCVA deposition is pulsed at $1 \mathrm{~Hz}$. For each pulse, an arc is initiated between the gold cathode and the trigger electrode, creating a dense plasma between the cathode and anode. After being filtered, the plasma is accelerated towards the sample, held at a bias of $2.5 \mathrm{keV}$. The gold ions are doubly charged, and the ion energy varies from $5 \mathrm{keV}$ at the beginning of the pulse to $50 \mathrm{eV}$ at the end of the pulse. The implantation process is explained in detail in references 26 and 27. This procedure is repeated twice at a $40^{\circ}$ angle to create mirror image electrodes within the fluidic channel as shown in Fig. 1(b). After the implantation, 4 gold electrodes are sputtered on the surface in the 4 corners of the implanted area to ensure good electrical contact to the implanted layer.

Then, we lithographically pattern an ITO-coated glass slide (30-60 ohm/square, Sigma Aldrich) to create electrical contact with the gold implanted region as well as provide electrodes for the open side of the microfluidic channel as shown in Fig. 1(c).
Spin-on-glass (Honeywell) is coated onto specific regions of the ITO-coated glass slide to insulate the electrodes for the microfluidic channel from the gold ion implanted region of the PDMS. Inlet and outlet ports are punched into the PDMS with a holepunching machine and the PDMS chip is given an oxygen plasma treatment for $20 \mathrm{~s}$ at $50 \mathrm{~W}$. Immediately afterwards, the PDMS chip is bonded to the ITO-coated glass slide. The finished device is shown in Fig. 1(a).

The finished chip contains 8 electrodes on the ITO. Half of the electrodes are used to manipulate particles within the microfluidic channel directly from the ITO-coated substrate. The other electrodes are utilized to make contact with the electrodes inside the PDMS microfluidics. When the electrodes on the PDMS walls and on the ITO are utilized independently, the applied electric fields are primarily in a plane parallel to the substrate with the capability of producing two separate orthogonal electric fields. Using a combination of the ITO electrodes and PDMS electrodes, the applied electric fields can be orthogonal to the plane of the substrate. By separately controlling each orthogonal electric field in the 3 axes with 8 distinct electrodes, we gain great flexibility in specifying the direction and distribution of the electric fields inside the fluidic channel. Further structuring such as arbitrary sidewall angles and thinner electrodes may be necessary to obtain more arbitrary alignment of electric fields throughout the entire fluidic channel.

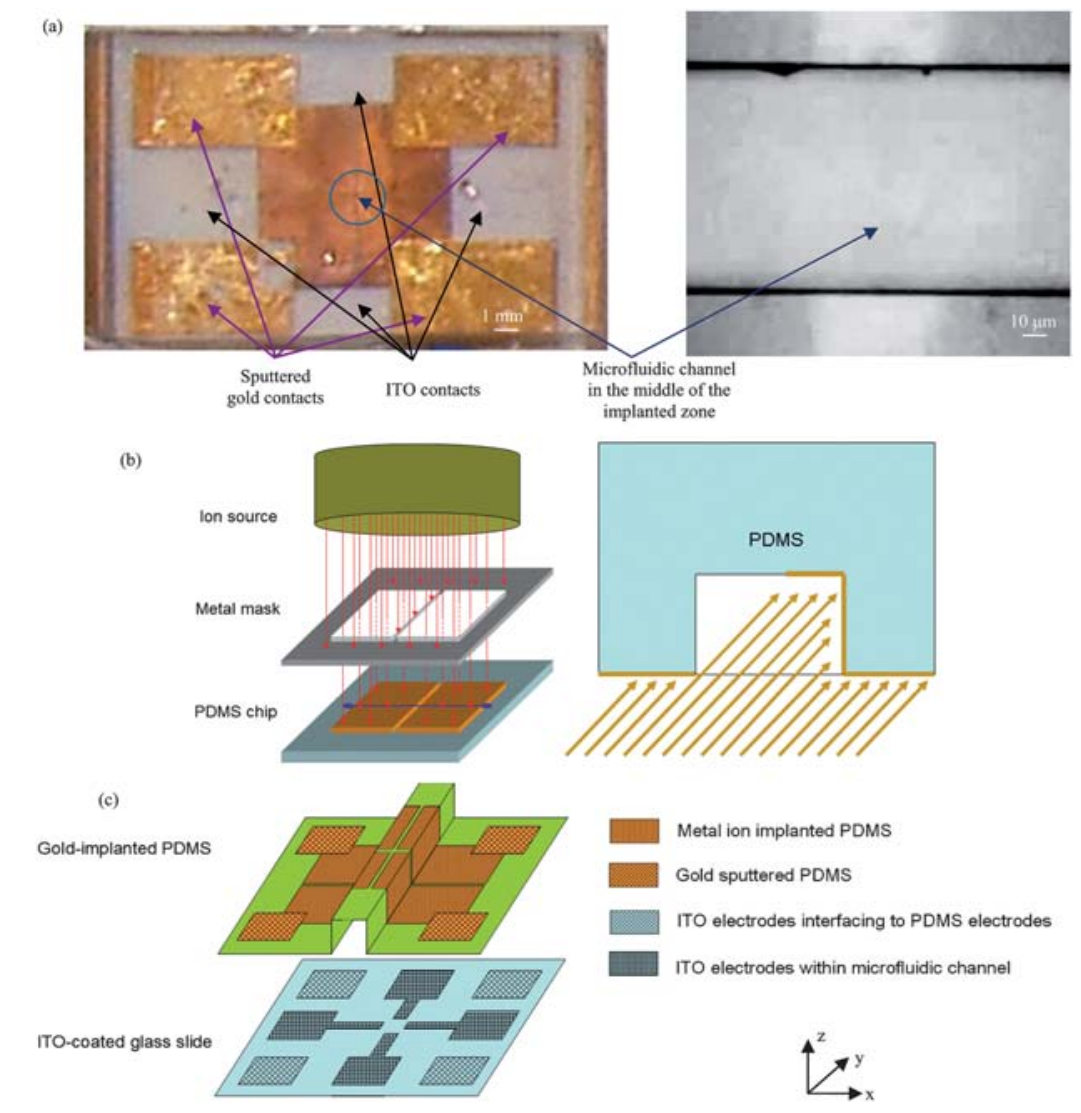

Fig. 1 Picture of the device. (a) The finished device and close up image. In both images, the microfluidic channel runs from left to right between the sputtered gold contacts. In the close up image, there are implanted gold electrodes on the left and right of the image. (b) The ions are implanted at an angle to create electrodes on the walls of the fluidic channel. (c) A lithographically patterned ITO-coated glass slide is bonded to the PDMS chip. Half of the electrodes on the ITO-coated glass slide connect to the PDMS electrodes. 
To apply the AC voltages to the electrodes, a function generator (HP 3312A) is utilized to generate frequencies between $1 \mathrm{~Hz}$ to $10 \mathrm{MHz}$ at voltages up to $10 \mathrm{~V}_{\mathrm{pp}}$. The output of the function generator is connected to a custom built circuit to control the electrode voltages through a set of relays with the USBcontrolled Velleman P8055. By selecting which set of electrodes is connected to either output of the function generator through the PC, we can either float the electrode or set it to be one of the active applicators of the electric field.

\section{Simulation}

We can investigate the field distributions generated by threedimensional structuring of electrodes within a fluidic channel through finite element simulations using the COMSOL software package. For a given electrode separation with only electrodes on the substrate, we note that the electric field distribution within the microfluidic channel is similar to the fringing fields of a capacitor. Depending on the height within the channel, the particle sees a significantly different magnitude of the $x$-axis component of the electric field. Particles closer to the electrodes sees at least one order of magnitude higher electric field than particles closer to the topwall of the microfluidic channel (for a $50 \mu \mathrm{m}$ channel height, a 10-fold change occurs when the particle's height is changed from $5 \mu \mathrm{m}$ to $45 \mu \mathrm{m}$ ). When the electrodes are coated on the PDMS, the magnitude of the $x$-axis component electric field seen by the particle is more uniform throughout the height of the fluidic channel.

The following parameters were utilized for the simulations: relative static permittivities of 2.8 for PDMS, ${ }^{28} 4.2$ for the glass slide (given by COMSOL) and 80.1 for water within the fluidic channel, ${ }^{29}$ with an ITO and gold ion implanted PDMS electrical conductivity of $2 \times 10^{5} \mathrm{~S} \mathrm{~m}^{-1}$. We obtain this value by assuming the ITO is $100 \mathrm{~nm}$ thick and the gold ion implanted PDMS is $50 \mathrm{~nm}$ thick while measuring the ITO sheet resistance to be $50 \mathrm{ohm} / \mathrm{square}$ and the gold ion implanted PDMS to be $100 \mathrm{ohm} / \mathrm{square}$. For the first set of simulations, we assume a $100 \mu \mathrm{m}$ wide by $70 \mu \mathrm{m}$ tall fluidic channel that is $300 \mu \mathrm{m}$ long. There are two electrodes on the top of the glass substrate that are spaced $100 \mu \mathrm{m}$ apart. The fluidic channel is placed on top of this gap. There are also electrodes on the PDMS in the same configuration as the actual implantation. Fig. 2(a) shows, at 3 different slices in height, the simulation of the electric field when only the electrodes on the substrate are utilized. Fig. 2(b) shows the simulation of the electric field with all the electrodes utilized. The uniformity of the magnitude of the $x$-axis component of the electric field can be compared in arrow plots on the $z=60 \mu \mathrm{m}$ plane for both figures. However, there is no uniformity in the magnitude of the $z$-axis component of the electric field.

For the second set of simulations, we take a cross section with the fluidic channel moving liquids in and out of the plane.

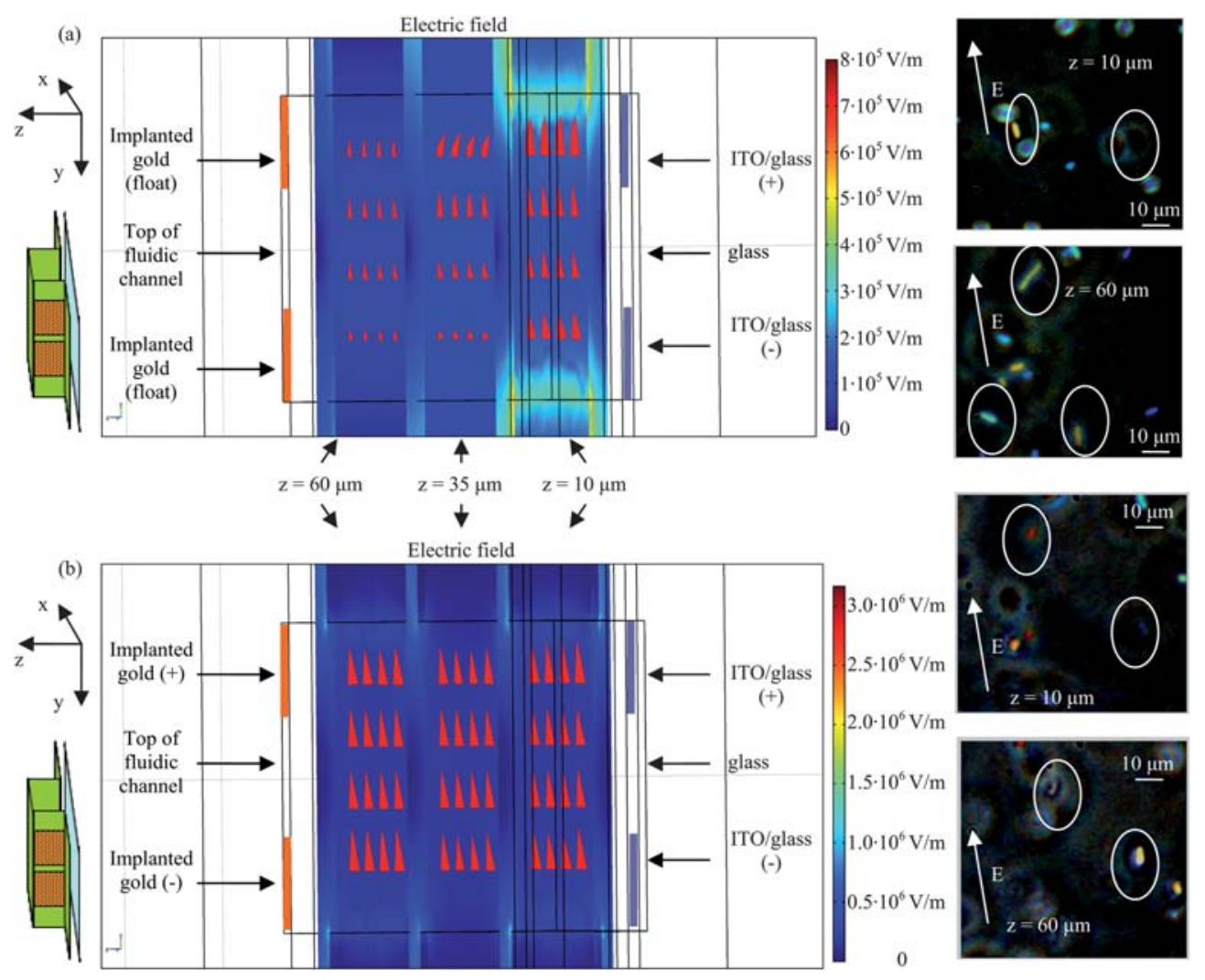

Fig. 2 Simulation of electric field within a fluidic channel. PDMS surrounds the microfluidic channel except on the right-hand side of the image where the ITO-coated glass slide is located. (a) With planar electrodes on ITO-coated glass slide substrate only. Images to the right show $E$. coli aligned at different $z$ planes. The ITO/glass is at $z=0 \mu \mathrm{m}$ and the top of the fluidic channel is at $z=70 \mu \mathrm{m}$. At $z=10 \mu \mathrm{m}$ all the bacteria are aligned. At $z=60 \mu \mathrm{m}$, the electric field does not orient all the bacteria. Some of the bacteria are on the substrate and do not move. They can be seen moving out of focus. (b) With electrodes on the microfluidic channel as well as the ITO-coated glass slide substrate. At both $z=10 \mu \mathrm{m}$ and $z=60 \mu \mathrm{m}$, all flowing bacteria are aligned. 
There is an electrode on the glass substrate as well as two electrodes on the walls of the PDMS. The gap between the two PDMS electrodes is $10 \mu \mathrm{m}$. We simulate the electric field generated orthogonal to the fluidic flow. Fig. 3(a) shows the simulation of the electric field for the direction parallel to the substrate. Fig. 3(b) shows the simulation of the electric field in the direction pointing from the substrate to the top of the fluidic chip. The electric field is not completely uniform throughout the microfluidic channel due to the coating of the top-wall of the device. This can be visualized in Fig. 3(c) by looking at the negative dielectrophoretic forces, which is proportional to the gradient of the electric field. There is a strong force pushing most of the particles in the channel experiencing negative dielectrophoretic forces down and towards the middle of the fluidic channel. Arrows on the plot indicate the magnitude and direction of the force. The contours indicate where the magnitude of the force is the same. The force is largest at the top and the two lower corners of the fluidic channels, becoming smallest at the bottom and the two top corners of the fluidic channels. For the two top corners, the forces are quite weak in the $z$-axis and we visualize few bacteria in this region experimentally.

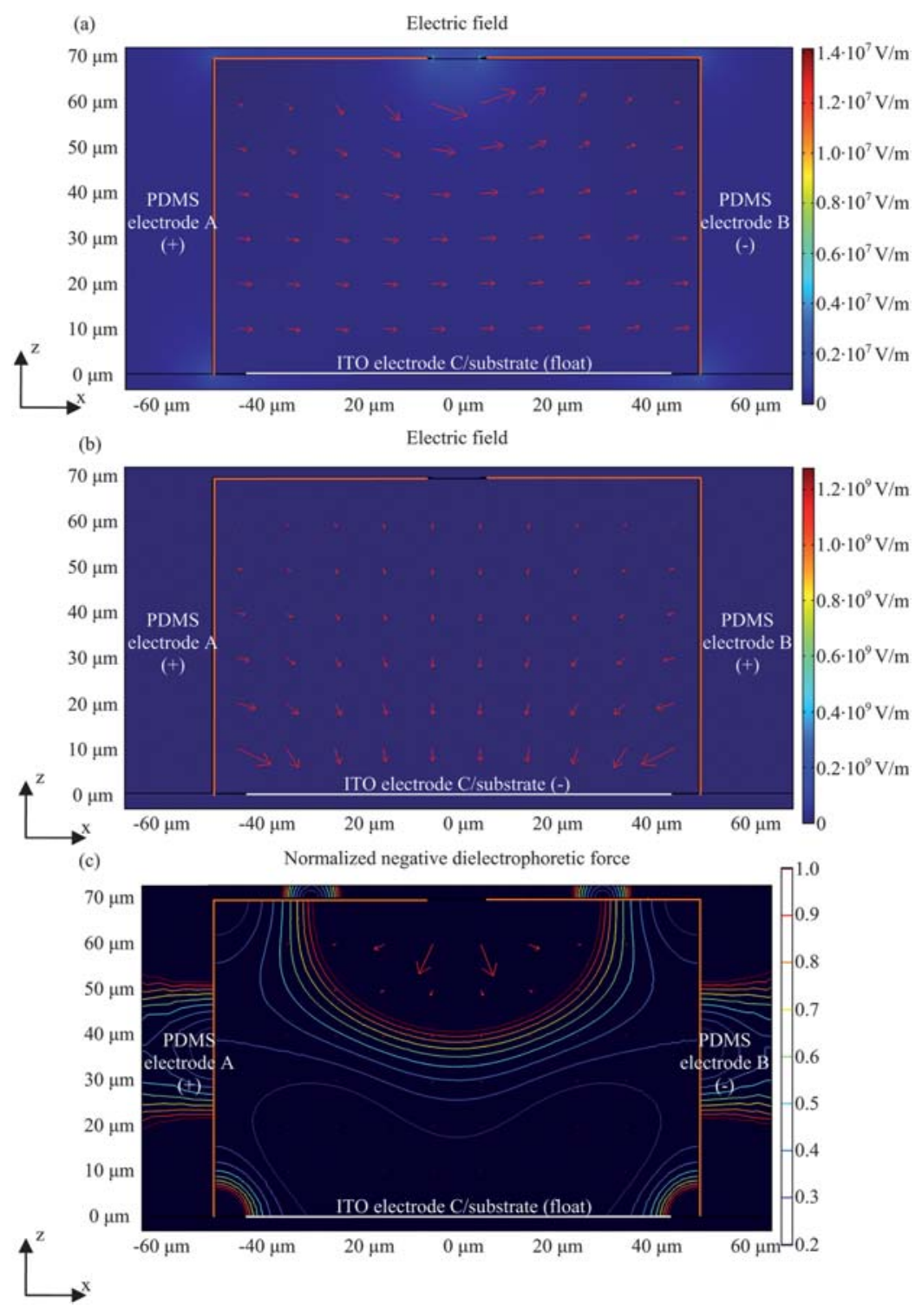

Fig. 3 Simulation of electric field and negative dielectrophoretic forces within a fluidic channel. PDMS is on top, the left and the right of the channel. ITO-coated glass is the substrate on the bottom. (a) The electric field is applied across the fluidic channel between the PDMS electrodes A and B. (b) The electric field is applied from electrodes on the fluidic channel to the ITO-coated glass slide between ITO electrode C and PDMS electrodes A and B shorted together. (c) Normalized negative dielectrophoretic forces experienced by a particle when the electric field is applied across the fluidic channel between PDMS electrodes A and B. Contour lines denote equivalent force magnitudes with the smallest forces represented in blue and the largest forces represented in red, going from $0.6 \times 10^{15} \cdot \alpha \mathrm{N}$ to $3.0 \times 10^{15} \cdot \alpha \mathrm{N}$ in steps of $0.3 \times 10^{15} \cdot \alpha \mathrm{N}$ where $\alpha=($ Volume of bacteria) $) \cdot \frac{\varepsilon_{\mathrm{m}}}{2} \cdot \operatorname{Re}\left\{\frac{\varepsilon_{\mathrm{p}}-\varepsilon_{\mathrm{m}}}{\varepsilon_{\mathrm{m}}{ }^{*}}\right\}, \varepsilon_{\mathrm{p}}$ is the dielectric permittivity of the particle, and $\varepsilon_{\mathrm{m}}$ is the dielectric permittivity of the medium. ${ }^{30}$ Arrows indicate magnitude and direction of this force. For negative dielectrophoresis, collection occurs in the area denoted by the contour with the smallest force indicated in blue. 


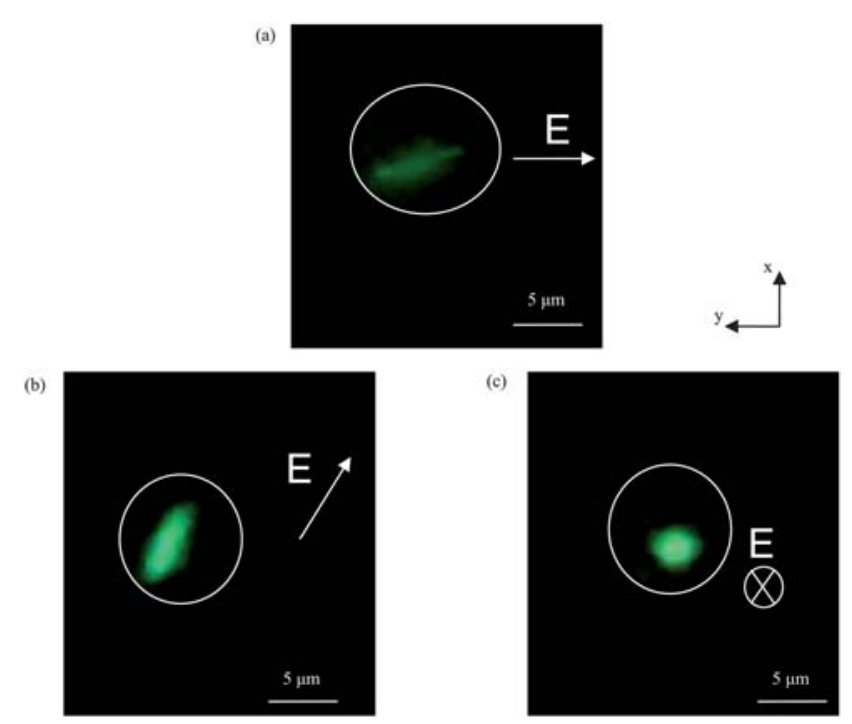

Fig. 4 Demonstration of bacteria orientation. The white arrows indicate the direction of the applied electric field. The SYTO 9 fluorescence labeled bacterium is also aligned in this direction. White circles mark live bacteria which are currently in the fluidic channel. (a) The electric field is applied in the horizontal direction. (b) The electric field is applied with both vertical and horizontal components. (c) The electric field is applied in and out of the image plane. Electrodes are not shown.

\section{Experiments}

\subsection{Electro-orientation within a fluidic channel}

Bacterial orientation within a microfluidic channel is possible through the electro-orientation force. This force is exerted on an asymmetric particle within a medium due to the application of an electric field. By applying an AC frequency where the particle has a dielectric permittivity higher than the medium, the particle will align along the field. ${ }^{14}$ We can align the bacteria to any of the axial directions by simply applying the electric fields along the proper electrodes. This type of measurement is useful, for example, when an asymmetric sample of interest is located at a known position and the precise height, width and length of the particle need to be determined. Also, it is possible to flow the particle over a sensing structure in a particular orientation if one is interested in a particular viewing angle of the sample of interest, for example, the flagellum. This technique may be especially useful when wanting to probe particular entities within a biological cell, in particular, to orient microtubules. ${ }^{31}$

We utilize the same parameters as have been shown in the electro-orientation techniques of $E$. coli $\mathrm{K} 12$ described in previous work to align the bacteria to the electric field. ${ }^{17} 10 \mathrm{~V}_{\mathrm{pp}}$ at $10 \mathrm{MHz}$ is applied across two electrodes to obtain a preferred alignment. Live bacteria is flowed horizontally from right to left within the microfluidic channel at a rate of $20 \mu \mathrm{m} \mathrm{s}^{-1}$. Fig. 4(a) shows bacteria aligned to a horizontally applied electric field. Fig. 4(b) shows bacteria aligned to an electric field with both vertical and horizontal components. Fig. 4(c) shows bacteria aligned to an electric field which is applied in and out of the plane. Without an applied electric field, most bacteria prefer to align horizontally along the direction of flow.

\subsection{Dielectrophoresis within a fluidic channel}

Colloidal focusing within a microfluidic channel is possible through the dielectrophoretic force. This force is exerted on
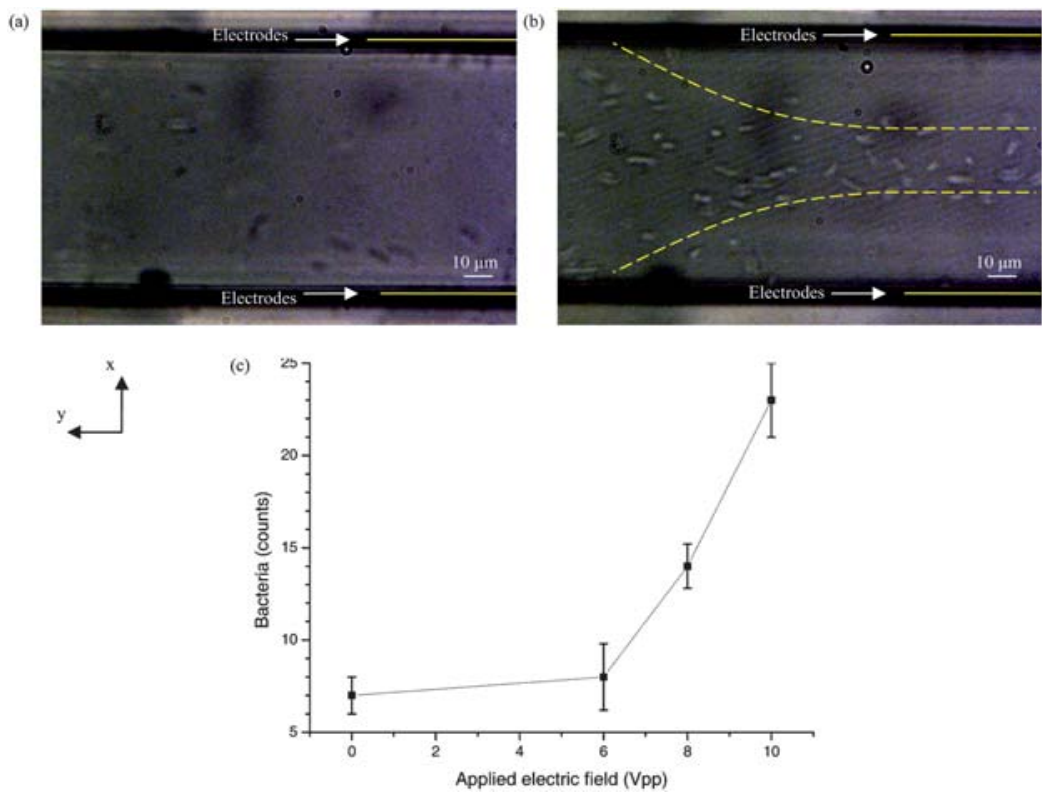

Fig. 5 Demonstration of bacteria concentration. The fluidic channel contains 4 electrodes in this picture with the bacterial solution entering from the right. An electric field can be applied on the right-hand side electrodes from the top of the image to the bottom of the image. (a) The electric field is not applied. Bacteria come into the picture from the right without preference for focusing within the fluidic channel or focal plane. (b) The electric field is applied on the noted right hand side electrodes. Bacteria come into the picture from the right in the center of the fluidic channel at the same focal plane. Note that the electric field is not applied on the left side electrodes. The bacteria immediately redisperse in both focal plane and within the fluidic channel as marked by the dashed lines. (c) Data for applied electric field strength versus bacteria within the central $25 \mu \mathrm{m}$ of the fluidic channel. The measurement of the position was taken for a total of 25 bacteria each time. Voltages lower than $4 \mathrm{~V}_{\mathrm{pp}}$ resulted in a similar profile to no electric field. 
a particle within a medium due to the application of a nonuniform electric field. By utilizing an AC frequency where the particle has a dielectric permittivity lower than the medium, the particle will move towards where the fields are weakest. ${ }^{13,22} \mathrm{We}$ utilize this force to move particles to the middle of the rectangular microfluidic channel. This is advantageous where a sample of interest must be flowed over a region which is much smaller than the size of the fluidic channel. This can be especially useful for sparse suspensions as the colloids will be concentrated in the middle of the region. A key advantage of this approach over fluidic flow focusing is the ability to flow colloidal solutions at a slower rate without losing any focusing capability. This may be especially useful for lab-on-a-chip applications that combine larger microfluidic channels with smaller structures.

We change the conductivity of the solution to $170 \mu \mathrm{S} \mathrm{cm}^{-1}$ and apply a frequency of $1 \mathrm{MHz}$ to obtain negative dielectrophoresis. $10 \mathrm{~V}_{\mathrm{pp}}$ is applied across the two PDMS sidewall and topwall electrodes. Bacteria is flowing horizontally from right to left within the microfluidic channel at a rate of $10 \mu \mathrm{m} \mathrm{s}^{-1}$. Fig. 5(a) shows no field applied. Fig. 5(b) shows bacteria in the center of the fluidic channel due to the field being applied on the electrodes to the right-hand side. This concentration occurs as the bacteria flow along the channel from the sidewall electrodes. Fig. 5(c) shows the bacteria counts in the central $25 \mu \mathrm{m}$ of the fluidic channel across different electric field strengths. With $10 \mathrm{~V}_{\mathrm{pp}}$, most of the bacteria are being concentrated to within $25 \mu \mathrm{m}$ of the center of the fluidic channel. This corresponds to an increase in the concentration by about 15 times. This is similar to the concentration of microbeads and human leukemia cells with $10 \mathrm{~V}_{\mathrm{pp}}$ at $10-100 \mathrm{kHz}$ in. ${ }^{22}$

\section{Conclusion}

Three-dimensional electrode patterning within a PDMS chip for microfluidic application utilizing metal ion-implantation is described for the first time. The ease of electrode structuring without the need for several fabrication steps highlights the advantages of utilizing this particular technique for electrical manipulation in lab-on-a-chip applications.

\section{References}

1 G. M. Whitesides, Nature, 2006, 442, 368-373.

2 D. Psaltis, S. R. Quake and C. Yang, Nature, 2006, 442, 381-386.
3 M. A. Unger, H. P. Chou, T. Thorsen, A. Scherer and S. R. Quake, Science, 2000, 288, 113-116.

4 T. Thorsen, S. J. Maerk1 and S. R. Quake, Science, 2002, 298, 580584.

5 D. Erickson and D. Li, Anal. Chim. Acta, 2004, 507, 11-26.

6 C. Yamahata, M. Chastellain, V. K. Parashar, A. Petri, H. Hofmann and M. A. M. Gijs, J. Microelectromech. Syst., 2005, 14, 96-102.

7 A. A. Darhuber, J. P. Valentino, S. M. Troian and S. Wagner, J. Microelectromech. Syst., 2003, 12, 873-879.

8 H. A. Stone, A. D. Stroock and A. Ajdari, Annu. Rev. Fluid Mech., 2004, 36, 381-411.

9 D. A. Boyd, J. R. Adleman, D. G. Goodwin and D. Psaltis, Anal. Chem., 2008, 80, 2452-2456.

10 M. Krishnan, J. Park and D. Erickson, Opt. Lett., 2009, 34, 19761978.

11 B. Y. Park and M. J. Madou, Electrophoresis, 2005, 26, 3745-3757.

12 L. Wang, L. A. Flanagan, E. Monuki, N. L. Jeon and A. P. Lee, Lab Chip, 2007, 7, 1114-1120.

13 T. B. Jones, IEEE Eng. Med. Biol. Mag., 2003, 22, 33-42.

14 R. D. Miller and T. B. Jones, Biophys. J., 1993, 64, 1588-1595.

15 P. Gascoyne, C. Mahidol, M. Ruchirawat, J. Satayavivad, P. Watcharasit and F. F. Becker, Lab Chip, 2002, 2, 70-75.

16 L. Yang, P. P. Banada, M. R. Chatni, K. S. Lim, A. K. Bhunia, M. Ladisch and R. Bashir, Lab Chip, 2006, 6, 896-905.

17 J. W. Choi, A. Pu and D. Psaltis, Opt. Express, 2006, 14, 9780 9785.

18 A. Jamshidi, P. J. Pauzauskie, P. J. Schuck, A. T. Ohta, P.-Y. Chiou, J. Chou, P. Yang and M. C. Wu, Nat. Photonics, 2008, 2, 86-89.

19 W. Choi, S. W. Nam, H. Hwang, S. Park and J. K. Park, Appl. Phys. Lett., 2008, 93, 143901.

20 Z. X. Lu, L. F. Wood, D. E. Ohman and M. M. Collinson, Chem. Commun., 2009, 4200-4202.

21 C. Iliescu, G. L. Xu, V. Samper and F. E. H. Tay, J. Micromech. Microeng., 2005, 15, 494-500.

22 C. Yu, J. Vykoukal, D. M. Vykoukal, J. A. Schwartz, L. Shi and P. R. C. Gascoyne, J. Microelectromech. Syst., 2005, 14, 480-487.

23 K. P. Nichols, J. C. T. Eijkel and H. J. G. E. Gardeniers, Lab Chip, 2008, 8, 173-175.

24 D. C. Duffy, J. C. McDonald, O. J. A. Schueller and G. M. Whitesides, Anal. Chem., 1998, 70, 4974-4984.

25 R. W. Stark, M. S. Stalder and A. Stemmer, Microelectron. Eng., 2003, 67-68, 229-236.

26 S. Rosset, M. Niklaus, P. Dubois and H. Shea, Sens. Actuators, A, 2008, 144, 185-193.

27 S. Rosset, M. Niklaus, P. Dubois and H. R. Shea, Adv. Funct. Mater., 2009, 19, 470-478.

28 Polymer data handbook 2nd edition, editor J. E. Mark, Oxford University Press, New York, NY 2009.

29 CRC Handbook of Chemistry and Physics 89th edition, CRC Press, Boca Raton, FL 2008.

30 T. B. Jones, Electromechanics of Particles, Cambridge University Press, Cambridge, UK 1995.

31 I. Minoura and E. Muto, Biophys. J., 2006, 90, 3739-3748. 